\title{
Quelques résultats à l'appui d'une hypothèse de MM. Danel, Durand et Condolios sur le phénomène de la saltation
}

\author{
Some results in support of a hypothesis \\ on the phenomenon of saltation
}

\author{
par L. ESCANDE
}

MEMBAE DE L'INSTITUT, INGENIEUR I.E.T., DIRECTELR DE L'E.N.S.K.H.T.

\begin{abstract}
Le sillage d'un obstacte en régime transitoire reproduit les configurations successives qui caractérisent les régimes permanents relatifs à des nombres de Reynolds croissants. Ce résultat mis en évidence en étudiant le sillage d'un cylindre circulaire montre le bien fondé de l'hypothese fondamentale de MM. Danel, Jurand et Condolios au sujel du phénomène de saltation (Introduction à l'étude de la saltation, 1i) Houille Blanche, $n^{\circ}(;, 1953)$.
\end{abstract}

Au cours de la séance du 18 juin 1953 du Comité Technique de la Société Hydrotechnique de France, MM. Danel et Durand ont présenté une belle analyse du phénomène de saltation, basée sur un ensemble d'observations très judicieuses. Un point fort intéressant nous a paru résider dans l'explication du fait qu'un caillou, au moment où il se détache du fond et se trouve enveloppé par le courant, fait souvent un saut, parfois très important, sous l'effet d'une brusque mise en vitesse. MM. Danel et Durand attribuent cette action particulièrement énergique du courant au fait que, pendant le régime transitoire présidant au contournement du gravier par le fluide, le sillage ne se forme que progressivement, de telle sorte qu'au début la force d'entrấnement possède une valeur nettement supérieure à celle que l'on observerait, dans l'écoulement permanent du liquide, avec la même vitesse d'attaque, autour du grain maintenu immobile.

Cette remarque très importante de MM. DANEL et DURAND concorde parfaitement avec les résultats obtenus par M. Camichel dans ses observations sur la rétrogradation des particules à l'aval d'un obstacle et dans ses recherches effectuées en collaboration avec MM. Baubiac et Crausse, sur le régime transitoire d'écoulement autour d'un obstacle, en régime laminaire et en régime turbulent.

\begin{abstract}
Disturbance of un obstacle in transitory regime reproduces the successive profiles which characterize permanent regimes for increasing Relynolds numbers.

This result, which is obtained by studying the disturbance of a circular cylinder, shows that it is based on the fundamental hypothesis eslablished by Messrs. Danel, Durand and Condolios on the subject of saltation (Introduction to the study of saltation. la Houlle Blanche, $\left.n^{\mathrm{n}} 6,1953\right)$.
\end{abstract}

En particulier, M. Bavbiac (1) a effectué une comparaison systématique des valeurs de la résistance d'un cylindre circulaire, en régime permanent et en régime transitoire. Ses expériences l'ont amené à conclure que, pendant le règime transitoire, la résistance est, à chaque instant, supéricure à la valeur qu'elle aurait, en régime permanent, pour la même vitesse à l'infini; dans certains cas en particulier, et au début du régime transitoire la résistance peut être doublée et mème triplée.

II nous a paru intéressant de compléter ces études en filmant, au moyen d'un appareil de prises de vues à cadence rapide, le sillage d'un cylindre en régime transitoire.

Le cylindre, d'un diamètre de $2 \mathrm{~cm}$, est mont: dans le plan de symétrie d'un ajutage à section droite rectangulaire, de $20 \mathrm{~cm}$ de largeur sur $5 \mathrm{~cm}$ de hauteur.

L'ouverture extrêmement rapide d'un robinet aval permet de faire varier quasi instantanément le débit de l'ajutage.

Le cylindre est creux et deux fentes, placées sur la partie amont, comme l'indique la fi-

(1) J. Baubiac: Etude expérimentale, en régime transitoire, du sillage et de la résistance des corps immergés. Thèse d'ingénieur-docteur de la Faculté des sciences de Toulouse, 1936 . 
gure 1, permettent d'alimenter le sillage, au moyen d'une solution de nitrate d'argent renfermant de la poudre daluminium, sans perturber l'écoulement.

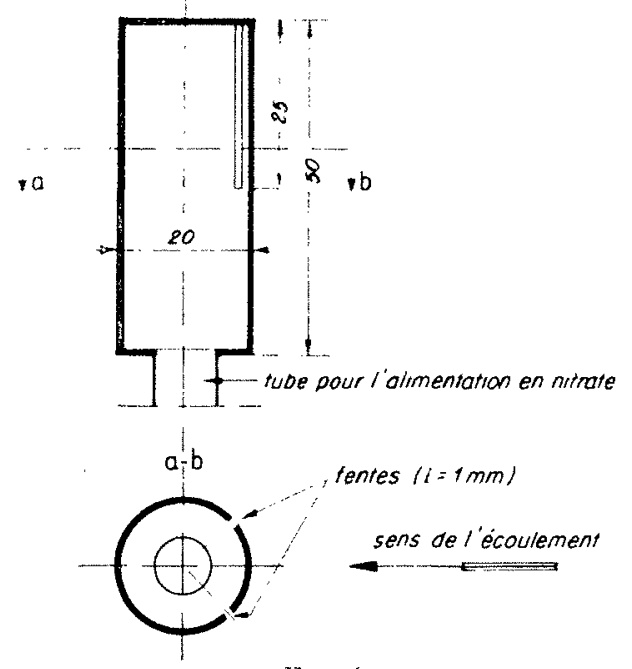

Fig. 1

Les films obtenus mettent nettement en évidence la succession, pendant le régime transitoire, des configurations que l'on observe, derrière le cylindre, lorsqu'en régime permanent on ítudic le sillage pour des valeurs successives croissantes du nombre de Reynolds :
- Contournement du cylindre sans décollement (spectre analogue à celui de la solution de première espèce rencontrèc dans le cas d'un fluide parfait).

- Décollement et double tourbillon symétrique de sillage non turbulent (courbes $\alpha, \beta$ de M. Camichel).

-.. Tourbillons alternés.

Il est à noter que cette succession de phénomènes s'observe, quel que soit le régime permanent initial, c'est-à-dire que celui-ci corresponde lui-même à un fluide immobile, à un sillage sans décollement, aux courbes $\alpha, \beta$ ou aux tourbillons alternés.

Les photographies de la figure 2 correspondent à une vitesse moyenne d'attaque, dans le régime permanent initial, égale à $0,0133 \mathrm{~m} / \mathrm{s}$ et à la limite d'existence des courbes $\alpha, \beta$, lors de l'apparition des premières ondulations du sillage : dans le régime permanent final, la vitesse moyenne d'atlaque est de $0,188 \mathrm{~m} / \mathrm{s}$ et le sillage est caractérisé par les tourbillons alternés.

Dans le cas de la figure 3 , le régime final demeure le même, mais on part d'une vitesse initiale d'attaque de $0,0267 \mathrm{~m} / \mathrm{s}$ pour laquelle existent déjà les tourbillons alternés.

Dans les deux cas, on observe nettement, comme première conséquence de l'ouverture, un étranglement progressif du sillage initial, met-

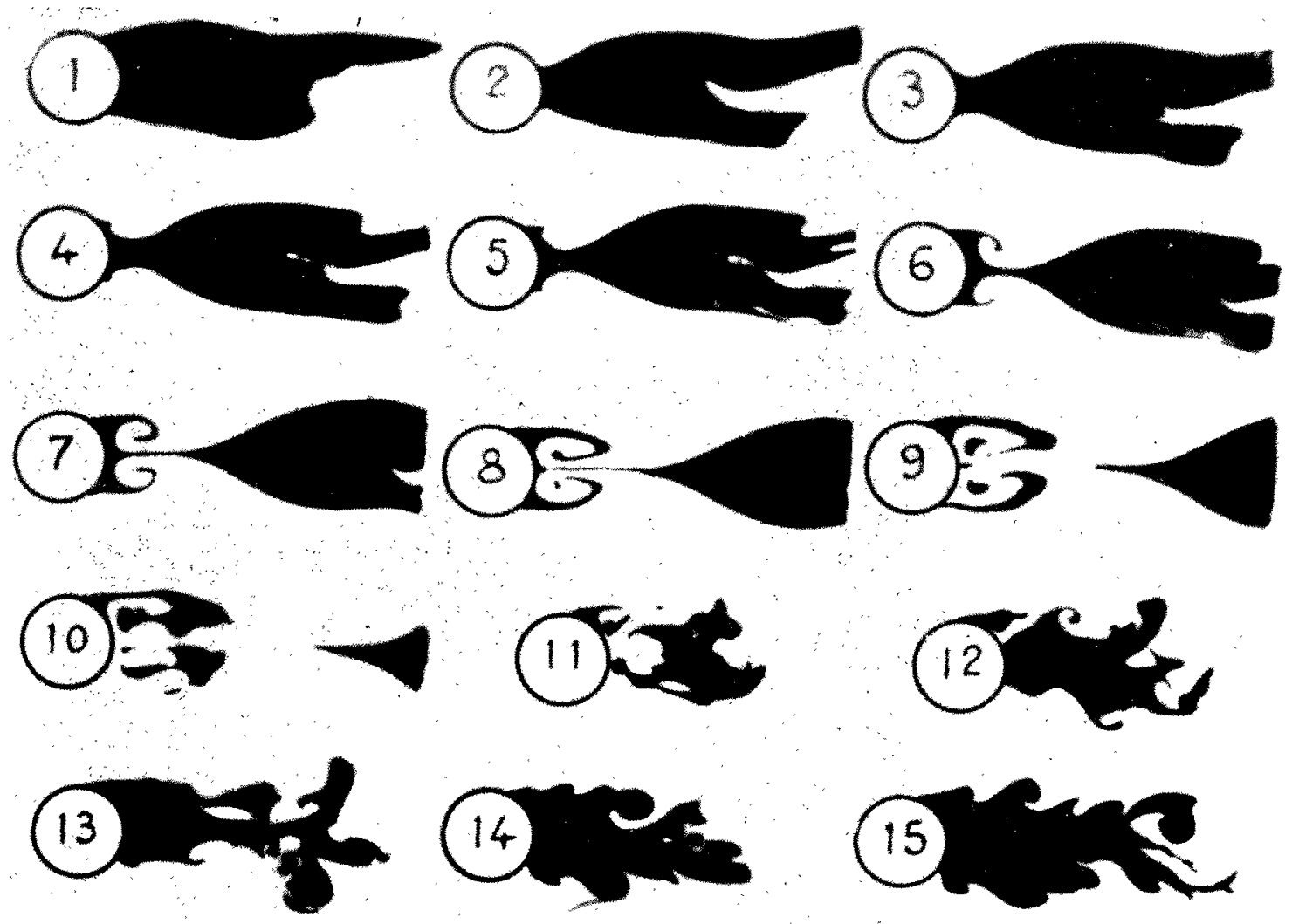

Fig. 2 

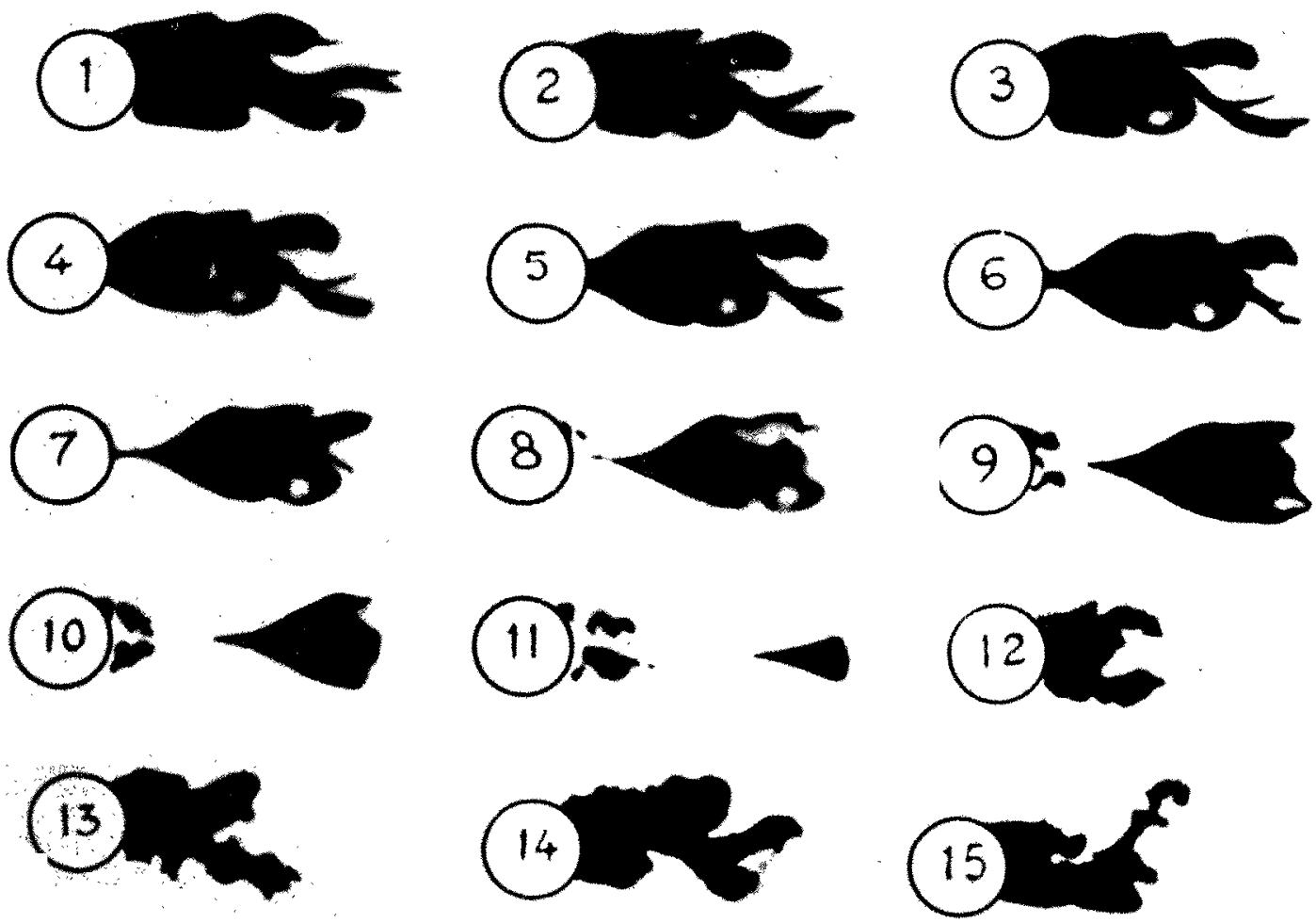

Fig. 3

Lant en évidence la solution de première espèce; on voit apparaître ensuite et se développer progressivement de l'amont vers l'aval, derrière le cylindre, les rouleaux caracléristiques des courbes $\alpha, \beta$ qui font place, à leur tour, aux tourbillons alternés, au bout d'un certain temps.

Les résultats ainsi obtenus sont relatifs à des conditions expérimentales qui ne peuvent se confondre avec celles dans lesquelles se produit le phénomène de saltation; elles en sont toutefois suffisamment voisines pour qu'on puisse en tirer des conclusions tout à fait favorables au bien fonde de l'hypothòse fondamentale de MM. DANEI. et DURAND.

\section{I S C US S I O N}

Présidenl: M. BanmLLoN

Sur la demande de M. le Président, M. Escanne précise que la vitesse du courant était variable et de l'ordre de $15 \mathrm{~cm} / \mathrm{s}$ : cela correspond à des nombres de Reynoms très faibles, et qui dépendent, d'ailleurs, de la dimension des grains.

M. le Président suggère de refaire ces intéressantes expériences avec un eylindre oscillant et rappelle que le temps nécessaire à l'établissement de la circulation autour d'un grain est différent selon que lon se place dans le plan ou dans l'espace à trois dimensions.

M. DanRreus remarque que l'impulsion donnée par la période variable et donnant la vitesse initiale parail très faible dans les hypothèses ordinaires, c'est-à-dira du grain isolé, et doit correspondre à la masse du fluide associé.

M. Escande précise que le grain immobile se trouve: soumis à la pleine vitesse du courant, ce qui provoque l'entrainement, mais que la vitesse relative du grain vis- a-vis de l'eau diminue ensuite, de lelle sorte que le grain retombe.

M. le Président suggèce aussi de filmer l'évolution des grains au voisinage de la paroi, où la vitesse est très faible : si on explique, d'abord, le décollement immédiat, on peut supposer que le grain va passer tapidement dans une région à vitesse beaucoup plus grande.

M. Darrieus demande s'il n'y aurait pas un effet. Magnus, dans le genre de celui que Von Kanmas avail mis en Evidence dans l'ascension des Montgolfieres.

M. le Président dit que l'effet Magnus ou le temps nocessaire a la circulation, à l'etablissement d'une couche limite a été étudié pour les plaques of les cylindres, mais non au voisinage des spheres on rotation, du moins à sa connaissance.

M. Darnaeus signale que Vos Kamman a decrit des expériences sur ces circulations.

M. Io Présidenl remercie M. Fscannes. 